\title{
Telescopic Overdenture-An Overlooked Treatment Modality for Partially Edentulous Patients: A Case Report
}

\author{
${ }^{1}$ Francis LL Fernandes, ${ }^{2}$ Sachin H Chaware
}

\begin{abstract}
Overdenture can be said to be the best example of preventive dentistry". When the clinical situation dictates 2 or 3 teeth (particularly canine and premolars) present on either side of the arch, particularly resorbed mandibular ridge, then Telescopic overdenture is considered to be the best option because it not only induces sufficient frictional retention but also helps the patient in easy placement and removal of the denture from the mouth. The main advantage of telescopic overdenture is that it transfers the forces along the long axis of the abutment teeth. This clinical case report describes a technique for telescopic overdenture. The telescopic design consists of 2 copings. The first coping is called primary coping was cemented onto the prepared abutment teeth. The primary coping is modified at the proximal cervical area by creating the frictional retention form in the form of grooves. The secondary coping, i.e. part of the metal framework engages the primary coping and subsequently generates the frictional retention at the proximo-cervical area. This design is technique sensitive, but aids in better retention, stability, support and proprioception which increases chewing efficiency and phonetics.
\end{abstract}

Keyword: Preventive prosthodontics, Retention, Stability, Telescopic overdenture.

How to cite this article: Fernandes FLL, Chaware SH. Telescopic Overdenture - An Overlooked Treatment Modality for Partially Edentulous Patients: A Case Report. J Contemp Dent 2018;8(3):148-152.

Source of support: Nil

Conflict of interest: None

\section{INTRODUCTION}

In this modern era, implant dentistry is gaining widespread attention in every clinical situation, and conventional prosthodontic treatment is no longer preferred by most, who enter the dental office. ${ }^{1}$ The practitioner must select the prosthodontic treatment which is not only clinically best but economical to the patients. Glossary of Prosthodontic terms (1999) defines overdenture as, "Any removable dental prosthesis that covers and rests on one or more remaining natural teeth, the roots of natural teeth, and/or dental implants; a dental prosthesis that

\footnotetext{
${ }^{1}$ Postgraduate Student, ${ }^{2}$ Professor

1,2Department of Prosthodontics and Crown and Bridge, MGV's KBH Dental College and Hospital, Nashik, Maharashtra, India

Corresponding Author: Francis LL Fernandes, Postgraduate Student, Department of Prosthodontics and Crown and Bridge, MGV's KBH Dental College and Hospital, Nashik, Maharashtra, India, e-mail: francisfernandes0288@gmail.com
}

covers and is partially supported by natural teeth, natural tooth roots, and/or dental implants." 2

Overdenture is the best example of preventive dentistry. ${ }^{3}$ Apart from proprioception, it plays an important role in mandibular positional sensibility. Overdenture have several advantages such as it improves the masticatory efficiency, enhances retention and stability that gives psychological benefit to the patient. Overdenture has many designs that include simple short and long copings, various ball and socket attachments, stud attachments and bar attachments. Overdenture is indicated in patients with few remaining teeth. It plays a significant role in in patients with congenital and acquired defects of the maxilla and mandible, transitional overdentures, immediate overdentures, remote overdentures, and removable partial overdentures.

Telescopic overdenture, is a double coping type of overdenture that selected primarily for the patient who is having multiple teeth present bilaterally, particularly mandibular arch. The primary coping present on the abutment teeth and secondary coping present in the denture framework. Both copings generate a frictional resistance thereby increasing the retention.

In 1887, The American System of Dentistry was reported first time about telescoped "bridgework". Schweitzer ${ }^{4}$ describe the telescoped lower removable partial denture over eight vital lower natural teeth. Ralph Boos reported the use of an upper denture telescoped over a patient's natural teeth in making a facial rehabilitation. Yalisove $^{5}$ discussed the theory of crown and sleeve coping retainers for removable partial dentures. The telescoping overdenture provides adequate retention and well accepted by the patient. The only requirement that it should have adequate fit between the primary and secondary copings, as it not only generates the frictional retention but it dictates the path placement and removal of the prosthesis.

This case report elicits the fabrication of a telescopic overdenture opposing a complete maxillary denture.

\section{CASE REPORT}

A 56-year-old male patient reported to the Department of Prosthodontics, MGV's KBH Dental College and Hospital, Nashik, Maharashtra, India with a complaint of difficulty in chewing and swallowing of food, because of completely edentulous maxillary arch and partially edentulous mandibular arch. Past dental history reveals that the teeth were lost due to severe decay and subsequent 


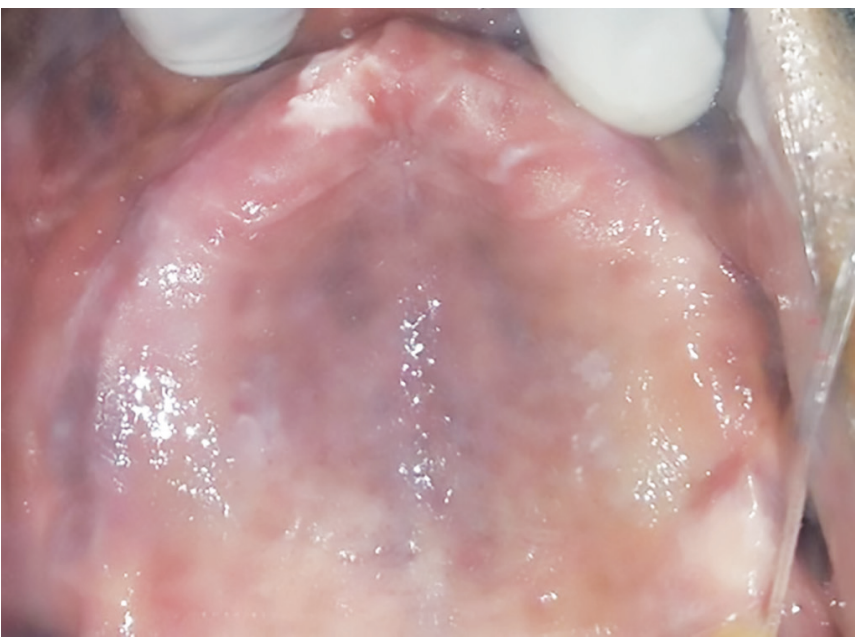

Fig. 1: Completely edentulous maxillary alveolar ridge

periapical infection. On clinical examination, the maxillary ridge is completely edentulous. The residual alveolar ridge is well rounded with sufficient height and width (Fig. 1). The mandibular arch is partially edentulous with $33,34,35$ and 44,45 teeth were present (Fig. 2). Clinically the remaining teeth of the mandibular arch show no pocket depth and no decay was noted. Radiographically, the bone was well-corticated and well-defined around the roots of the remaining teeth. Anteriorly, the edentulous ridge is well rounded and the posterior ridge is narrow and low well rounded.

Maxillary and mandibular diagnostic impressions were made. Diagnostic jaw relation was recorded followed by evaluation of interarch space. The interarch space of $13 \mathrm{~mm}$ was measured after diagnostic mounting (Fig. 3). Two treatment modalities were taken into consideration. Initially, Implant Fixed prosthesis was decided for both the maxillary and mandibular arch as the ridge condition was sufficient to place the implant and subsequent implant prosthesis. The economic condition and age of the patient were not to the favor for implant prosthesis. The second treatment plan was maxillary complete denture, and mandibular telescopic overdenture was planned. Cast partial denture was not taken into consideration as a treatment modality as few remaining teeth were present, and the framework would cause excessive torquing forces that would be detrimental to the attachment apparatus of the teeth present. Consequently, the complete denture was planned for the maxillary arch so that the occlusion can be very well executed in the artificial teeth.

\section{Clinical Steps}

The diagnostic impressions were made using irreversible hydrocolloid impression material for the mandibular arch (Tropicalgin; Zhermack, Italy) and medium fusing impression compound for the maxillary arch(Y-Dent India Ltd.). The casts were prepared using type III dental stone (Ultrastone; Kalabhai India Private Ltd.).The maxillary border molding was carried out with a low

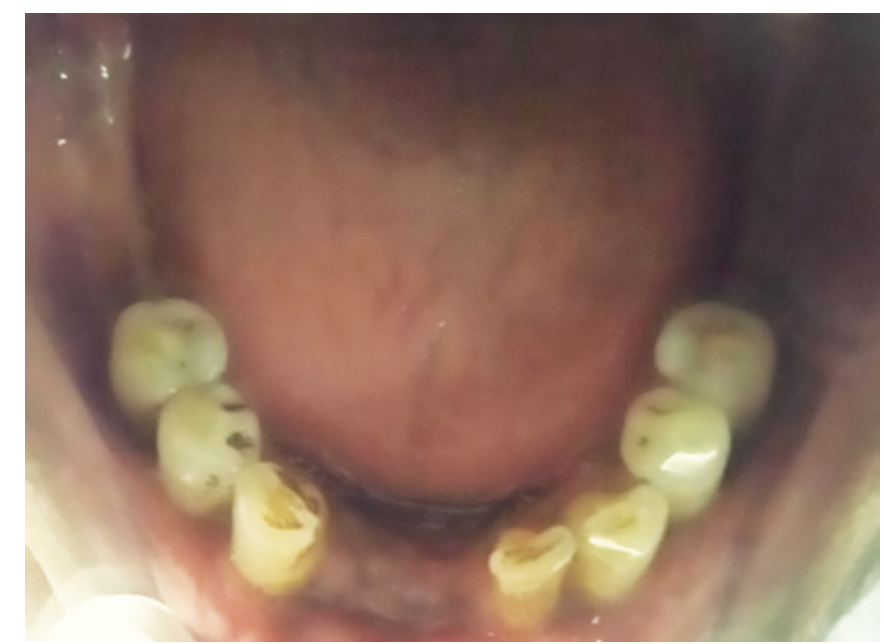

Fig. 2: Partially edentulous mandibular alveolar ridge

fusing compound and wash impression was made using light bodied elastomeric impression (Kerr Pvt. Ltd.). The impressions were poured with type III dental stone and the master cast was prepared for the maxillary arch. A tentative jaw relation was recorded and diagnostic mounting was done to evaluate the available inter-arch space.

Endodontic treatment was carried out for the remaining abutment teeth. Preparation of the abutment teeth was carried out for primary copings (Fig. 4) and the impression was made with elastomeric impression materials (Kerr India Private Ltd). The primary copings were fabricated with nickel-chromium alloy (Fig. 5). The primary copings were cemented to the abutment teeth by using a temporary luting cement (Kerr, Temp-bond) and a pickup impression was made so that secondary copings along with framework will get a precision fit with the primary copings. The secondary copings and framework were fabricated with cobalt chromium alloy (Fig. 6). The primary copings were permanently cemented on the prepared abutment teeth using a type I glass ionomer luting cement (GC type I cement; Luting and Lining Cement; GC Corporation Tokyo, Japan) (Fig. 7).

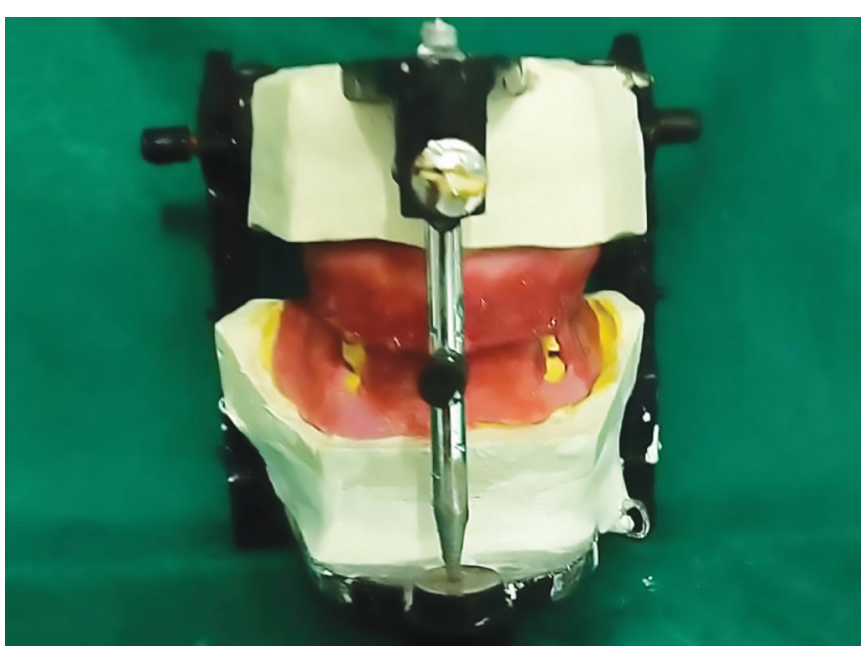

Fig. 3: Diagnostic jaw relation and diagnostic mounting 


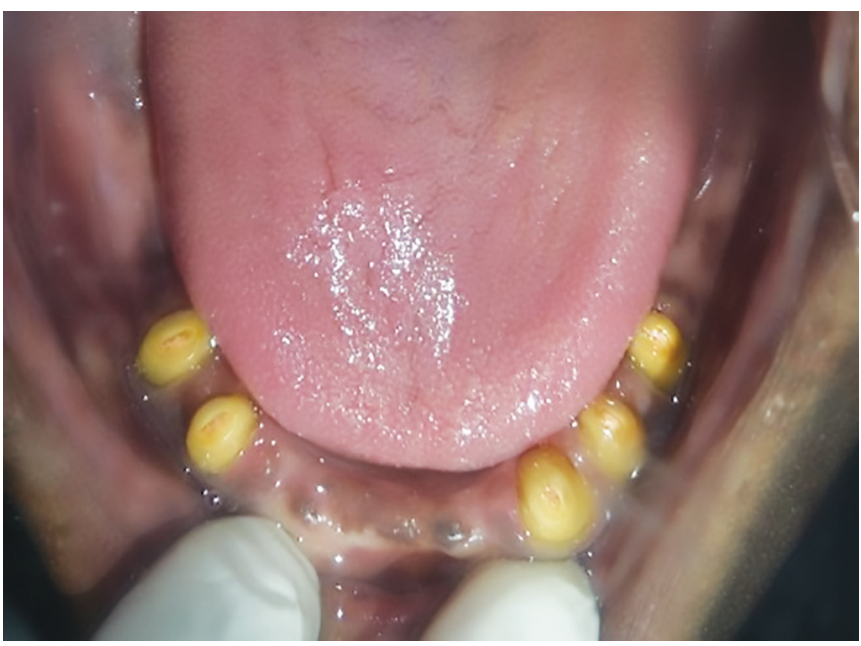

Fig. 4: Tooth preparation of abutment teeth to receive primary copings

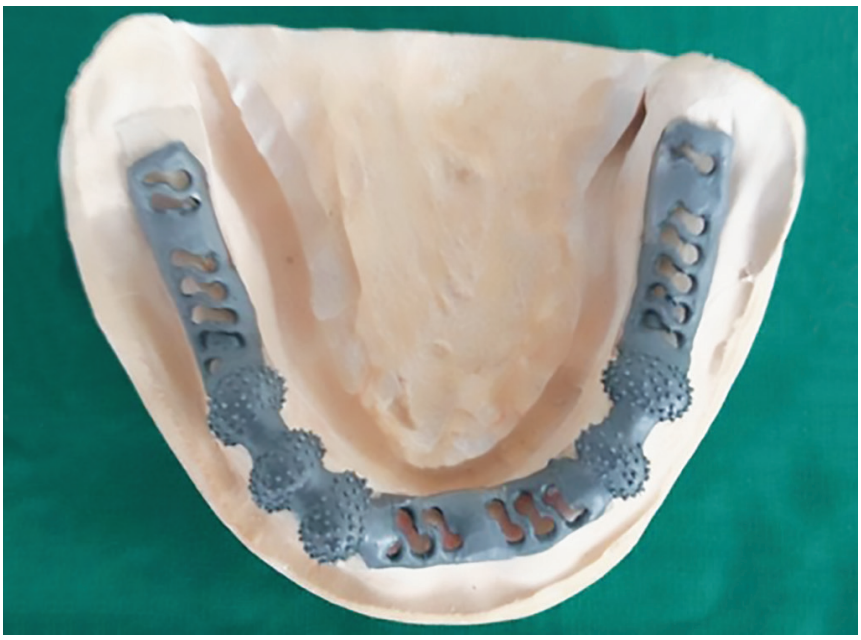

Fig. 6: Secondary copings and framework

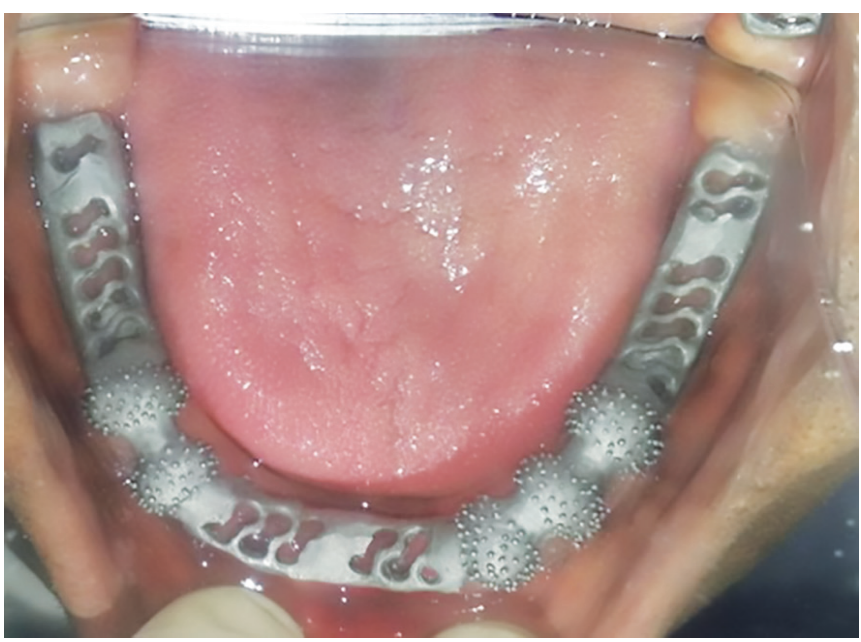

Fig. 8: Telescopic superstructure tried intraorally

The fit of the telescopic superstructure was checked intra-orally (Fig. 8). Jaw relations along with Face-bow record was recorded (Fig. 9). Try-in was done to verify the jaw relation, esthetics and phonetics (Fig. 10) which was followed by acrylization with heat-polymerized acrylic resin (Trevalon; Dentsply India Pvt Ltd., Guru-

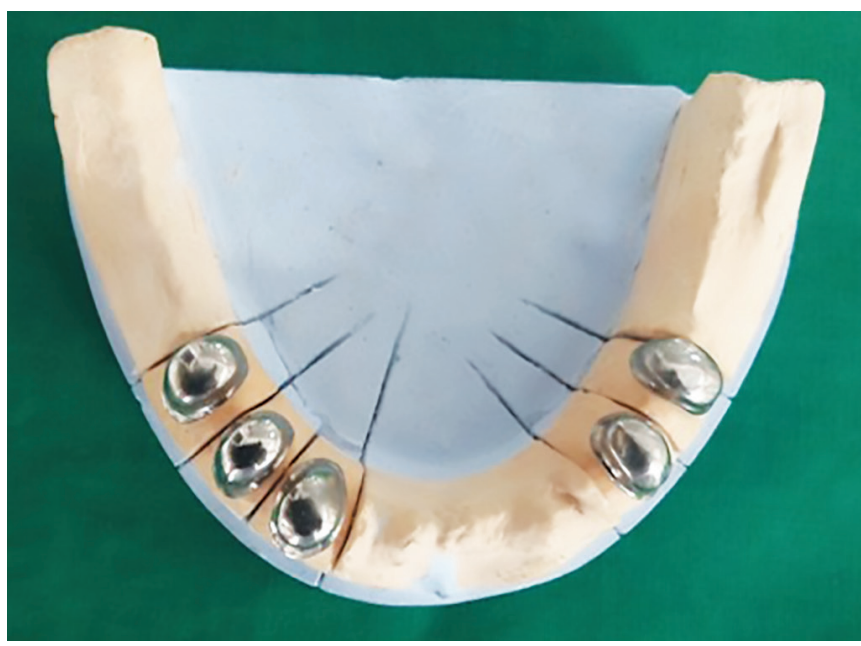

Fig. 5: Primary copings

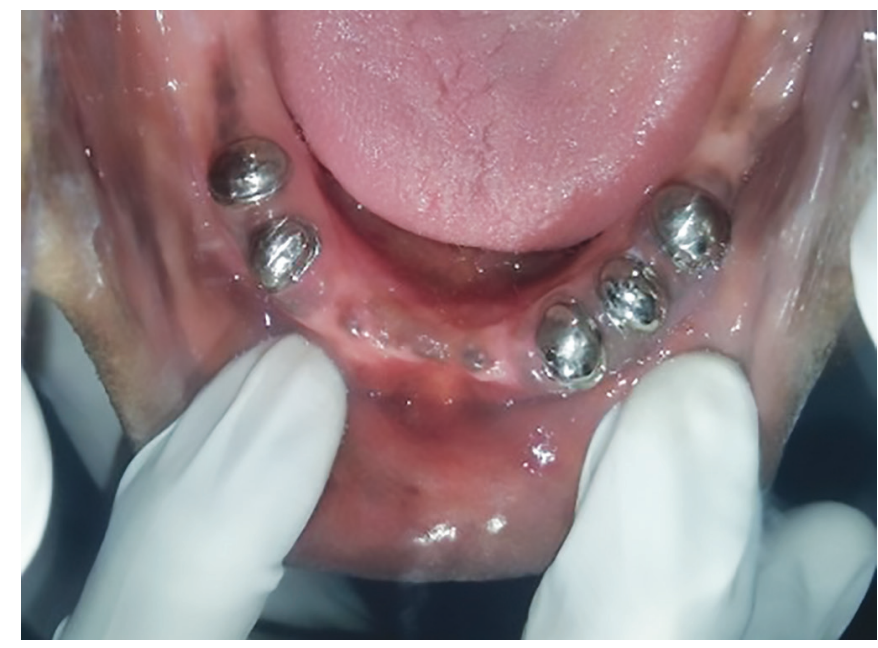

Fig. 7: Primary copings cemented intraorally

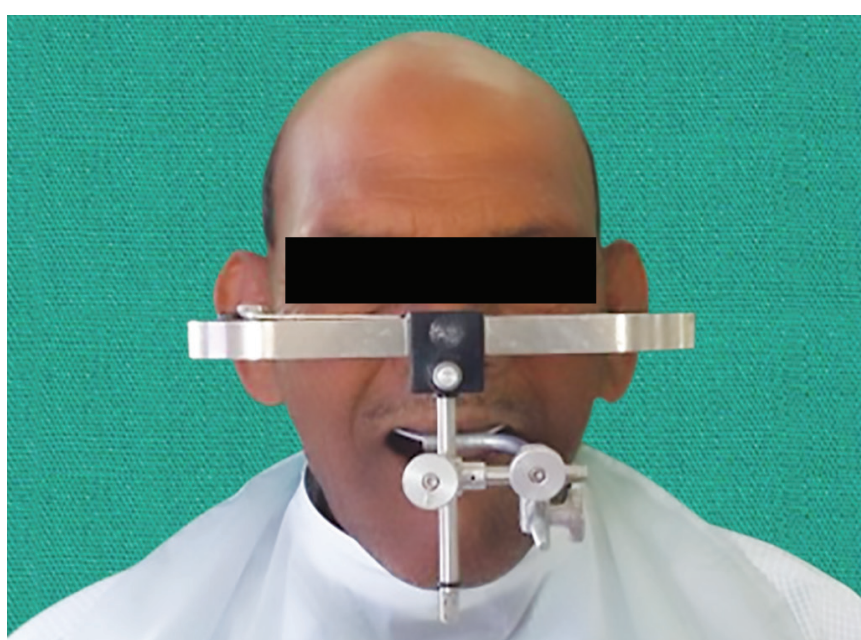

Fig. 9: Jaw relations along with Face-bow record

gram, Haryana, India) (Fig. 11). Finally, Denture Insertion was done (Figs 12 and 13) and the necessary friction fit desired was obtained. Oral hygiene instructions were given and the patient was intimated about the periodic and regular check-ups for the maintenance of the prosthesis. 


\section{DISCUSSION}

Miller ${ }^{6}$ stated that the maxilla and mandible were designed to house the teeth and not to support artificial dentures. For any patients; young, middle-aged or elderly, whose mental and physical condition are in equilibration, any therapy which can improve form, function, increase
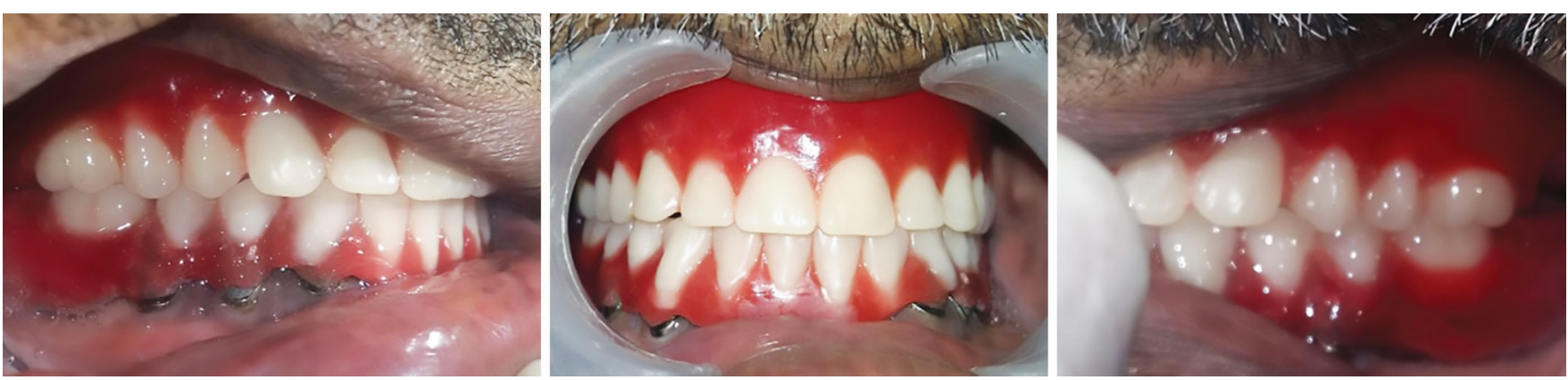

Fig. 10: Denture Try-in

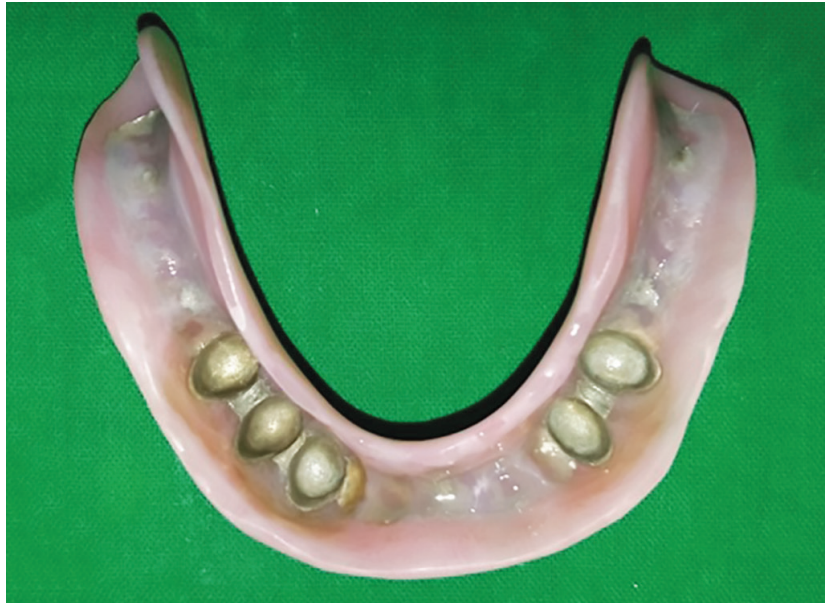

Fig. 11: Acrylization of mandibular overdenture using heat cure acrylic resin
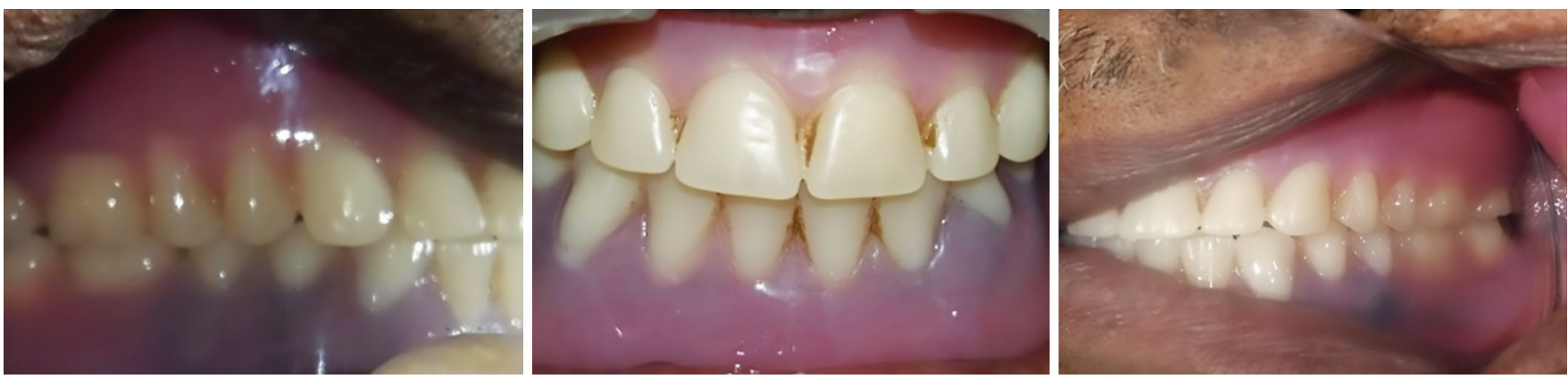

Fig. 12: Intraoral clinical photographs after denture insertion

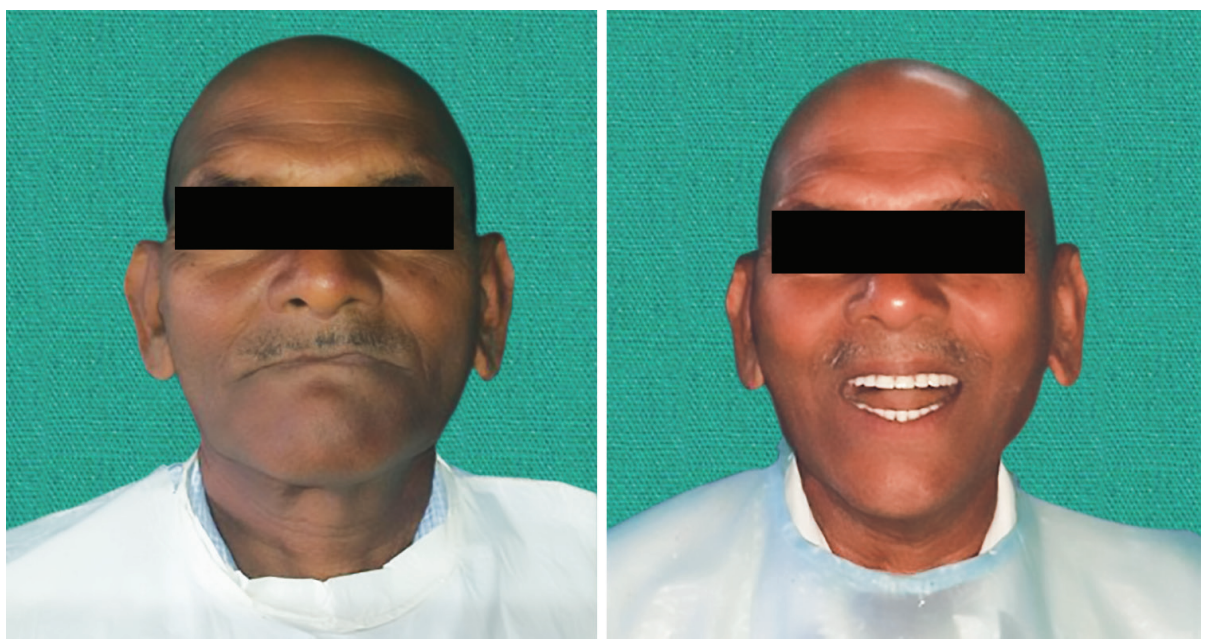

Fig. 13: Pre-operative and post-operative extraoral clinical photographs 
longevity as well as preserve the oral and perioral soft and hard tissues deserve a well-planned and organized treatment plan.

The patient in the present case report can be considered as an ideal candidate for telescopic overdenture as he was systemically healthy, had teeth present bilaterally with adequate bone support, no temporomandibular joint disorders and no allergy to base metal alloys. Crum and Rooney ${ }^{7}$ discussed the 5-year study of mandibular overdenture, found that retention of mandibular canines for overdentures led to the preservation of alveolar bone.

The main advantage of a telescopic overdenture is that it gives the psychological satisfaction of the presence of teeth in his/her mouth. Proprioception through the presence of periodontal fibers of the remaining natural dentition of the telescopic overdenture enhances patient's ability of mastication as compared to that of conventional complete denture. ${ }^{8}$ The prosthesis utilizes the basic concepts of the tooth-supported complete denture. The prosthesis is thus used as a therapeutic modality rather than a mere replacement of missing teeth.

Sufficient interarch space is an ideal requisite for this treatment modality to have a sufficient denture base thickness to avoid fracture, for the functional and aesthetic arrangement of the teeth and to have sufficient interocclusal distance. ${ }^{8}$ For this reason, a diagnostic jaw relation was recorded to evaluate the inter-arch space. Following which short primary copings were planned and root canal treatment of the abutment teeth was performed since exposure of pulpal tissue was a possibility.

The telescopic framework was cast in cobalt chromium alloy with retentive beads over the teeth for retention of acrylic. The framework helps in better stress distribution not only along the long axis of the teeth but also along the residual alveolar ridge. Also, a single path of placement and removal would be possible, thus enabling better manipulation skills.

\section{CONCLUSION}

Telescopic overdenture may be considered as a good alternative to conventional removable partial dentures if fabricated with utmost clinical expertise by a dentist along with meticulous oral hygiene maintenance by the patient. The preventive prosthodontic concept of telescopic overdenture gives the patient a gain in neuromuscular performance over his/her edentulous counterpart.

\section{CLINICAL SIGNIFICANCE}

Telescopic overdentures decreases the rate of residual ridge resorption. Also, it aids in better retention, stability, support and proprioception which increases chewing efficiency and phonetics of the partially edentulous patients.

\section{ACKNOWLEDGMENT}

Authors would like to acknowledge Dr Amruta Arun Joshi, Lecturer, Department of Periodontology, MGM Dental College and Hospital, Navi Mumbai, India.

\section{REFERENCES}

1. Kabcenell JL. Tooth-supported complete dentures. J Prosthet Dent 1971; 26(3):251-257.

2. The glossary of prosthodontic terms. J Prosthet Dent. 1999 Jan;81(1):39-110.

3. Preiskel, Harold W. Overdentures Made Easy: A Guide to Implant and Root Supported Prostheses. London: Quintessence Pub, 1996.

4. Schweitzer JM, Schweitzer RD, Schweitzer J. The telescoped complete denture: a research report at the clinical level. J Prosthet Dent. 1971 Oct;26(4):357-372.

5. Yalisove IL. Crown and sleeve-coping retainers for removable partial prosthesis. J Prosthet Dent. 1966 Nov-Dec;16(6):10691085.

6. Miller PA. Complete dentures supported by natural teeth. Tex Dent J. 1965 Sep;83:4-8.

7. Crum RJ, Rooney GE Jr. Alveolar bone loss in overdentures: a 5-year study. J Prosthet Dent. 1978 Dec;40(6):610-613.

8. Lord JL, Teel S. The overdenture: patient selection, use of copings, and follow-up evaluation. J Prosthet Dent. 1974 Jul;32(1):41-51. 